\title{
The role of vibration in the tactual perception of roughness
}

\author{
SUSAN J. LEDERMAN \\ Queen's University at Kingston, Kingston, Ontario, Canada \\ and \\ JACK M. LOOMIS and DEBORAH A. WILLIAMS
University of California at Santa Barbara, Santa Barbara, California
}

\begin{abstract}
Katz (1925) has argued that the sense of vibration underlies the tactual perception of roughness. However, Taylor and Lederman (1975) have suggested that vibration serves only to prevent the cessation of mechanoreceptor activity. In an experimental evaluation of these positions, it is shown that, although prior (selective) adaptation of the fingertip strongly affects the perceived magnitude of supraliminal vibrotactile signals, it fails to alter the perceived roughness of metal gratings. The results thus favor the Taylor and Lederman position. The paper also speculates on roughness coding by the mechanoreceptor populations present in glabrous skin of the human hand.
\end{abstract}

When a person moves his or her hand across a surface, vibrations are produced within the skin. In his untranslated monograph, The World of Touch (for English summaries, see Krueger, 1970, 1981), Katz (1925) argued strongly for the necessity of such vibrations in the perception of surface texture, or "modifications of the surface," as he described it. "For Katz, ... the vibration sense determines the modifications of the surface" (Krueger, 1970, p. 339). Katz concentrated mainly on the perception of surface roughness, one of the most prominent aspects of texture.

Taylor and Lederman (1975) have also argued for the importance of vibration in the tactual perception of surface roughness, but only as a means of preventing the cessation of activity in the relevant mechanoreceptor population(s). Their interpretation focuses, rather, on the contribution of various static characteristics of the skin deformation, for example, depth of penetration, volume of skin deformed, skin tension, stretch, etc.

If, as Katz argued, the vibration sense does underlie the perception of texture (or, more particularly, rough-

This research was conducted at the University of California at Santa Barbara while the first author was on sabbatical leave. Reprint requests should be sent to S. J. Lederman, Psychology, Queen's University, Kingston, Ontario K7L 3N6, Canada. This research was supported by NSERC Grant A9854 to the first author and NINCDS Grant 1 R01 NS 15129 to the second author. We would like to thank R. T. Verrillo and S. Bolanowski for their generous advice concerning equipment and for providing calibration curves of contactor displacement as a function of frequency for the vibrator used in these experiments. We would also like to thank Dan MacIntosh and Chick Hebert for their technical assistance. The late Bernard Klock of the Institute for Sensory Research at Syracuse University produced the contactor used in these experiments. ness), one must predict that the temporal frequency of vibration, which differentially affects the perception of vibration (e.g., Talbot, Darian-Smith, Kornhuber, \& Mountcastle, 1968; Verrillo, 1968), should also affect the perception of surface roughness. Both Katz (1925) and Lederman (1974) have found little effect of hand speed on the perception of roughness. Such results suggest either little contribution of fundamental pulse frequency and/or, perhaps, a speed constancy effect. The speed constancy interpretation appears to be unlikely in view of the fact that very similar results were subsequently obtained when the same gratings were moved across stationary fingertip skin at several very different speeds (Lederman, Note 1). However, Lederman (1973; Note 1) and Lederman and Taylor (1972) have provided additional psychophysical evidence against the notion that the temporal pulse frequency of impulses created by relative motion between skin and surface determines the perceived roughness of linear metal gratings. In one experiment, the fundamental pulse frequency was held constant by varying the duty cycle and thus also the groove and/or ridge width of the gratings. The wide variation in perceived roughness indicated that fundamental pulse frequency could not be the contributing factor. Conversely, when the temporal pulse frequency was varied by altering the ridge width and/or duty cycle (groove width was constant), the perceived roughness was unaffected. Overall, the results suggested that groove width was a major determinant of roughness perception, while fundamental pulse frequency, fundamental spatial period, duty cycle, and ridge width were all relatively unimportant.

In the current experiment, we chose an alternative approach, that is, selective vibrotactile adaptation, to 
evaluate the role of vibration in the perception of roughness. It is argued that if the sense of vibration does, in fact, underlie the perception of roughness, then a procedure that affects the perception of vibrotactile stimulation should also affect the perception of surface roughness. The next section provides the background behind our choice of technique.

Verrillo (1968) proposed a duplex model of mechanoreception for vibrotactile detection. He suggested that sensitivity to high-frequency stimulation (with maximum response at around $250-300 \mathrm{~Hz}$ ) is mediated by the Pacinian system (PC), while sensitivity to lower frequencies (particularly below about $40 \mathrm{~Hz}$ ) is mediated by a separate pathway with associated end-organs and referred to simply as the non-Pacinian system (NP). (More recently, Capraro, Verrillo, and Zwislocki, 1979, have suggested the participation of at least two nonPacinian receptor populations.) The psychophysical work has been reasonably well complemented by physiological data on glabrous skin of monkey obtained by Talbot et al. (1968). Their data show that the low and high branches of the human psychophysical threshold curves for vibration (as a function of frequency) correspond well to the frequency tuning curves of the QA (quickly adapting units presumed to end in Meissner corpuscles) and Pacinian afferents, respectively. More recently, masking and adaptation studies by Gescheider, Frisina, and Verrillo (1979), Labs, Gescheider, Fay, and Lyons (1978), and Verrillo and Gescheider (1976) add to the psychophysical evidence by demonstrating a fair degree of functional independence between the PC and NP systems. The adaptation studies by Gescheider et al. (1979) and Verrillo and Gescheider (1976), which pertain directly to the current study, showed that prior stimulation by an intense low-frequency signal $(10 \mathrm{~Hz})$ had little effect on the vibrotactile thresholds for high frequencies, while it raised the low-frequency thresholds in proportion to the intensity of the adapting stimulus. Selective adaptation to a high-frequency stimulus $(250 \mathrm{~Hz})$ raised the thresholds to high-frequency test stimuli as soon as the intensity of the adapting stimulus exceeded absolute threshold; however, it began to raise thresholds for a low-frequency test stimulus only when the intensity of the adapting stimulus was about $24 \mathrm{~dB}$ above threshold.

Selective adaptation effects have also been demonstrated at suprathreshold levels of vibrotactile stimulation. Hahn (1966) earlier used the selective adaptation technique to show a reduction in the subjective magnitude of a 34-dB SL adapting stimulus $(60 \mathrm{~Hz})$ over time. In the same report, he also demonstrated an even greater decrease in absolute vibrotactile sensitivity. Since Verrillo's theory has also been shown to account for suprathreshold vibration (Verrillo \& Gescheider, 1975), selective vibrotactile adaptation offers the possibility of differentially adapting the NP and PC systems at suprathreshold levels. By selectively adapting the skin to a low-frequency stimulus, one should in principle be able to diminish any contribution of the NP system. Any alteration in psychophysical response to roughness would presumably reflect the normal contribution of this system. Conversely, with adaptation to a high-frequency stimulus, any alteration in perceived roughness should reflect a corresponding contribution of the PC system. In the current study, a control condition was included in which the skin was adapted for a similar period of time to the stationary contactor.

The current paper is divided into three sections. In the first section, we describe the apparatus and pilot work that was performed both to arrive at an acceptable experimental setup and to choose the stimulus parameters. In the second section (Experiment 1), subjects judged the magnitude of suprathreshold test vibrations presented at both low and high frequencies following each of the three adaptation conditions described above. This study was designed to evaluate whether the perception of vibration was strongly (and selectively) affected by previous adaptation to a vibrotactile signal of a given frequency. In the third section (Experiment 2), subjects then judged the roughness of metal gratings following the same three selective adaptation conditions. If, as Katz would predict, the sense of vibration determines the perception of roughness, estimates of roughness should also be correspondingly affected by the three conditions of vibrotactile adaptation. The Taylor and Lederman model, however, predicts no change in roughness perception as a result of any selective vibrotactile adaptation procedure.

\section{APPARATUS, STIMULI, AND PRELIMINARY STUDIES}

\section{Apparatus}

Sinusoidal signals were produced by two Krohn-Hite 1000 function generators, each of which was calibrated by a HewlettPackard (Model 5211A) electronic counter. Timing of the test signal was controlled by a custom-built electronic switch and interval timer. The test stimulus was alternately switched on and off for 1-sec intervals; the rise-fall time was $100 \mathrm{msec}$. The test and adapting signals were passed through attenuators (Tech Laboratories, Model TA731) and then amplified (Amcron Model D75). A three-pole selector switch placed after the amplifier was used to permit switching between the adapting and test channels. The amplified signals were finally fed into a Ling (Model 203b) vibrator. Large-scale adjustments in signal intensity were produced with both the attenuators and amplifier; all fine tuning (including the determination of vibrotactile thresholds) was performed by adjusting the gain on the amplifier. An rms voltmeter was used to measure directly the input voltage to the vibrator. Subjects placed the distal pad of the right middle finger on a $1.3-\mathrm{cm}^{2}$ contactor mounted on the top of the vibrator. A large contactor area was chosen, as we wished to adapt the entire portion of the fingertip that would be used to feel the textured surfaces. Such a large contactor, of course, prevented the use of a surround. A felt-covered plate was set up as an armrest for the subjects. The contactor protruded through the armrest to a level of a few millimeters. The vibrator was mounted independently on a table that was physically separate from the other equipment and textured surfaces. This procedure helped to eliminate extraneous vibrations. No precise attempt was made to fix the depth of penetration of the contactor 
into the skin, since it was necessary for the subjects to move their fingers to and from the contactor each time a texture was presented. The subjects wore headphones into which was fed narrow-band noise $(20$ to $400 \mathrm{~Hz})$ to mask the sounds of the timing apparatus and vibrotactile signals.

The apparatus described above was used in all experiments reported in this paper.

\section{Textured Surfaces}

Twelve square-wave patterns were produced using photographic negatives of a square-wave pattern filmed at magnifications varying in equal steps of $4 \%$ of the original. The patterns were subsequently photoengraved on zinc plates. The dimensions of each plate were $6.4 \times 3.8 \times .16 \mathrm{~cm}$; the grating filled a $1.5 \times 1.5 \mathrm{~cm}$ area in the center. Groove width varied from .335 to $1.440 \mathrm{~mm}$ in average increments of $.100 \mathrm{~mm}$. The ridge wid th varied from .295 to $1.535 \mathrm{~mm}$ in average increments of $.110 \mathrm{~mm}$. Thus, spatial period ( 1 groove +1 ridge) varied from .630 to $2.975 \mathrm{~mm}$ in average increments of $.210 \mathrm{~mm}$.

\section{Vibrotactile Detection Thresholds}

The method of adjustment was used to obtain detection thresholds for the $20-$ and $250-\mathrm{Hz}$ adapting stimuli prior to adaptation. The subjects were required to adjust the gain on the amplifier until they could detect the presence of a vibration. The subjects began with a subthreshold setting, which varied from trial to trial to avoid adaptation during the actual measurement phase. Thresholds were measured as voltage input to the vibrator. The constant signal, rather than the pulsing envelope, was always recorded. From these threshold values, the millivolt values corresponding to various sensation levels were calculated and used to set intensity levels in the actual experiments.

\section{Pilot Studies for Experiments 1 and 2}

Reduced versions of Experiments 1 and 2 in their entirety were initially conducted with the three authors as subjects. In the pilot work, $10-$ and $250-\mathrm{Hz}$ signals at $40-\mathrm{dB}$ SL adaptation conditions and a static-adaptation control were used. In the static-adaptation condition, the middle fingertip rested lightly on the static contactor to control for possible effects due to a slight flattening of the skin (a physical deformation which was independent of temporal frequency effects). The pilot results were in reasonable accord with the major findings detailed in the next two sections. A number of changes subsequently introduced into Experiments 1 and 2, however, yielded somewhat cleaner results.

\section{Additional Pilot Work}

Subjects were instructed to exert a constant "light" force when examining the metal gratings. It was, of course, possible that perceived roughness might be affected by some consistent alteration in the force applied during the various adaptation conditions. Force was not controlled in these experiments, but is known (Lederman \& Taylor, 1972) to be an important determinant of perceived roughness. We therefore measured, independently of the actual experiments, the average force applied by each subject in each of the three adaptation conditions to metal gratings resting on a Mettler electronic balance. Each average was based on five measurements per plate for each adaptation condition/subject combination. The two most extreme gratings in the series were used. The applied force remained essentially unchanged in the three adaptation conditions. In Experiment 2 proper, the plates were mounted on a strain-gauge (Sensotec Model 31, 0-150 g) with output to a Daytronic strain gauge conditioner (Model 9170). The voltage from the conditioner was fed into a Beckman Type RS dynagraph to provide continuous on-line recording of the applied forces. Once again, however, there were no systematic differences among the three adaptation conditions.

\section{EXPERIMENT 1}

Experiment 1 was designed to test whether selective adaptation would affect the perceived magnitude of supraliminal vibration. According to the logic that underlies the current research, it was necessary to demonstrate a strong effect in this experiment before one could properly evaluate the role of vibration in the perception of roughness. A magnitude-estimation procedure was used rather than the subjective matching technique chosen by Hahn (1966). Subjects made estimates of the magnitude of sensations of vibrotactile test stimuli (at both 20 and $250 \mathrm{~Hz}$ ) that varied in intensity. The same set of judgments was required following selective adaptation to the static contactor." (control) and to both 20 - and $250-\mathrm{Hz}$ adapting stimuli presented at 40 and 45 (or 50 ) $\mathrm{dB}$ SL, respectively. The low-frequency test signal was raised from 10 to $20 \mathrm{~Hz}$, despite the fact that there would likely be less separation of the PC and NP systems. At $10 \mathrm{~Hz}$, one could not guarantee that the skin followed the contactor without the finger "riding" it. This in turn meant that the actual skin displacement could be less than the "nominal" value. Thus, the reader is cautioned to consider all displacement values reported in this paper as "nominal." As high an adapting intensity as possible was used (without overloading the circuit or being noxious to the subject) to further separate the PC and NP systems.

\section{Method}

Subjects. To reduce bias, three naive subjects participated in both Experiments 1 and 2. The subjects were young adults with no known abrasions or insensitive areas on their fingertips.

Procedure. Six detection thresholds were determined for both 20 and $250 \mathrm{~Hz}$, and the two averages subsequently were used as the 20- and $250-\mathrm{Hz}$ threshold values. The subjects learned to make free-modulus magnitude estimates of line lengths. They were instructed to assign a number (fraction, decimal, or whole number) that matched the perceived length of the line presented. Following the initial practice, they then made practice estimates of the magnitude of five different vibrotactile stimulus intensities, including 5 and $40 \mathrm{~dB} \mathrm{SL}$. The test stimuli varied in intensity from 5 to $40 \mathrm{~dB}$ SL in $5-\mathrm{dB}$ steps. Three test blocks (each consisting of the eight intensities) were presented at $20 \mathrm{~Hz}$, alternating with three blocks in which the test frequency was $250 \mathrm{~Hz}$. Order of presentation of the eight intensities within each block was quasi-random. The set of 48 trials was repeated after $10 \mathrm{~min}$ adaptation to the static, $20-\mathrm{Hz}$, and $250-\mathrm{Hz}$ conditions of adaptation. The intensity level of the $20-\mathrm{Hz}$ adaptation signal was $40 \mathrm{~dB}$ SL for all subjects. The levels for the $250-\mathrm{Hz}$ adaptation signal were 45,50 , and $50 \mathrm{~dB}$ SL for Subjects S, M, and D, respectively. Each subject repeated the three adaptation sets on a second day, so that adaptation blocks were completely counterbalanced across combinations of subjects and days. At the start of each trial, the subject was warned of the impending test stimulus. The experimenter switched from adaptation to test channel, allowing the stimulus to remain on for approximately $4 \mathrm{sec}$. The subject readapted for an additional $26 \mathrm{sec}$ following the presentation of each test stimulus. When needed, a small hairdryer was used to direct warm air toward the subject's finger while it was 
resting on the contactor to keep the skin from cooling. At least $1 \mathrm{~h}$ elapsed between adaptation conditions for each subject.

\section{Results}

Perceived magnitude of vibration (log geometric means of the magnitude estimates) and associated standard errors of the $\log$ values are plotted as a function of vibrotactile intensity for the three adaptation conditions (Figure 1). Each datum is based on six scores. The data for each combination of subject and test frequency are plotted separately. In general, perceived magnitude increases monotonically with increases in vibrotactile intensity. Of particular interest in the current experiment is the strong effect of selective adaptation that was demonstrated by each subject. Following adaptation to a $20-\mathrm{Hz}$ signal ( $40 \mathrm{~dB} \mathrm{SL})$, the perceived magnitude estimates of the $20-\mathrm{Hz}$ test stimuli markedly decreased (relative to the control); there was considerably less effect on the $250 \cdot \mathrm{Hz}$ test stimuli. The converse was also evident when the fingertip

\section{$20 \mathrm{HZ}$ TEST}

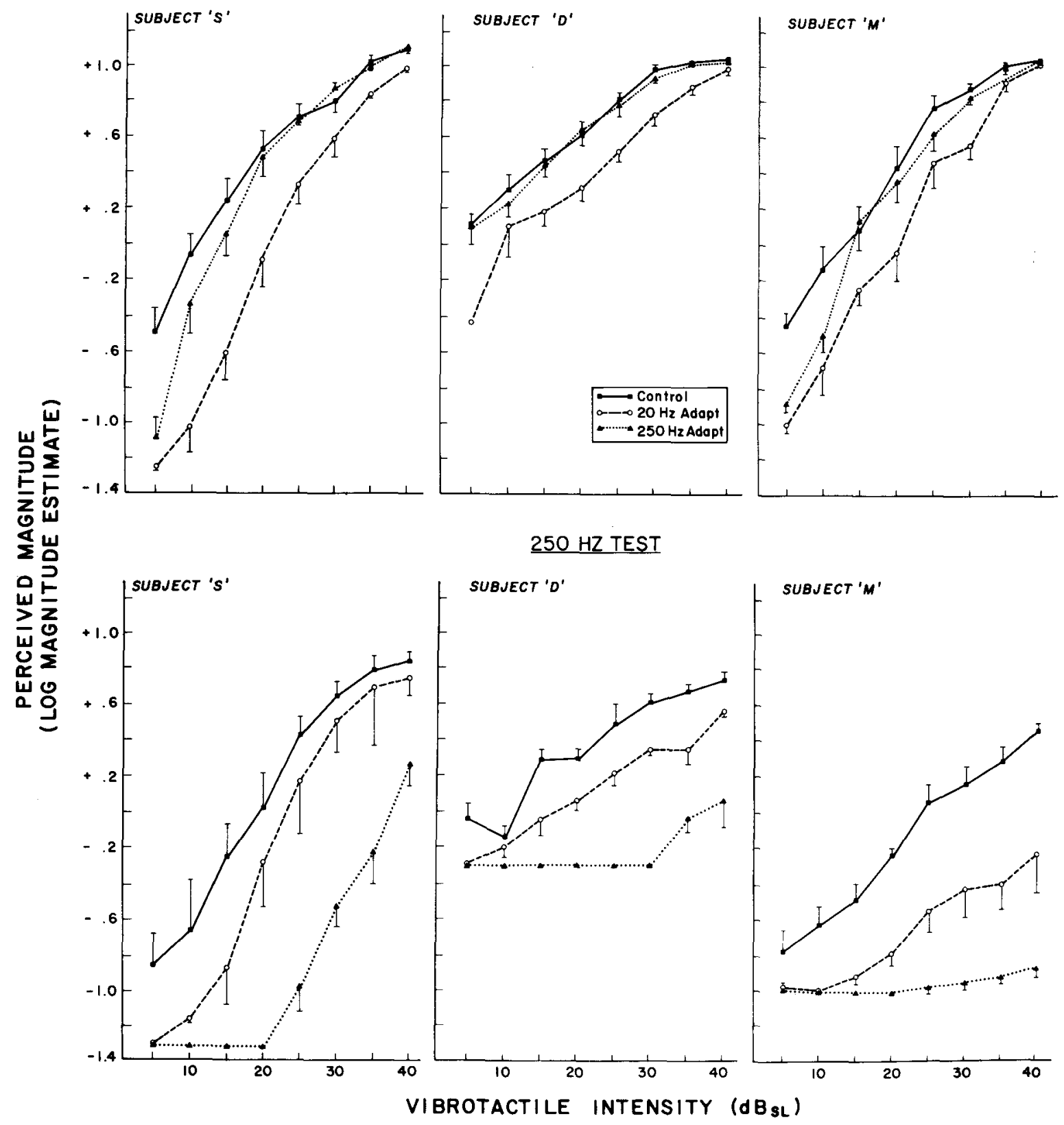

Figure 1. Experiment 1: Perceived magnitude of vibration and associated standard errors as a function of vibrotactile intensity and adaptation state (static, $20 \mathrm{~Hz}$, and $250 \mathrm{~Hz}$ ). The adapting intensity of the $20-\mathrm{Hz}$ signal was $40 \mathrm{~dB}$ SL for all subjects; the adapting intensity of the $250-\mathrm{Hz}$ signal was 45,50 , and $50 \mathrm{~dB}$ SL for Subjects S, M, and D, respectively. 
was adapted to an intense $250 \cdot \mathrm{Hz}$ vibrotactile stimulus; that is, there was markedly less reduction in the magnitude estimate of the $20-\mathrm{Hz}$ test signals than there was for the $250-\mathrm{Hz}$ test signals. Following adaptation to $250 \mathrm{~Hz}$, the subjects were unable to detect $250-\mathrm{Hz}$ test signals presented at a number of the lower intensities. Lastly, it may be noted that differential adaptation effects were most pronounced at the threshold end of the intensity range. This phenomenon was also evident in Hahn's data (1966).

In summary, the results of Experiment 1 indicate a large and consistent effect of selective vibrotactile adaptation on the perceived magnitude of vibrotactile stimulation.

\section{EXPERIMENT 2}

The next stage in the study was to evaluate the role of vibration in the perception of surface roughness by testing whether the latter was also affected by selective vibrotactile adaptation. Taylor and Lederman's (1975) model of roughness assumes that vibration per se does not contribute to roughness; the model, therefore, predicts no effect of selective adaptation. However, if vibration does underlie the perception of roughness, as Katz argued, then perceived roughness estimates should be affected by vibrotactile adaptation. Selective adaptation effects would presumably indicate differential contributions of the PC and NP systems which are thought to code vibration.

\section{Method}

Subjects. The same subjects participated in both experiments.

Procedure. The subjects made free-modulus magnitude estimates of the roughness of the 12 metal gratings described earlier. Practice with some of the plates preceded the actual test period. Each grating was presented a total of four times for each adaptation condition. The presentation order of the 48 stimulus con- ditions was random. Each subject repeated the 48 trials under control (static), $20-\mathrm{Hz}$, and $250-\mathrm{Hz}$ adaptation conditions. The intensity levels were the same as those used in Experiment 1. The three adaptation conditions were repeated in a second day's session. The order in which the adaptation conditions were presented was counterbalanced across the six combinations of subjects and days. Following the initial 10-min adaptation period, each trial consisted of the subject's moving his or her finger from the contactor to the plate, examining the surface for a maximum of $3 \mathrm{sec}$, and immediately returning the finger to the contactor (static or vibrating) for approximately $30 \mathrm{sec}$ of readaptation. The subjects were instructed to apply a constant, light force and to move their hands at any speed they chose.

\section{Results}

The data are shown for individual subjects in Figure 2. Log geometric means of the magnitude estimates are plotted as a function of log groove width for each of the adaptation conditions. Each datum is based on eight scores. Standard errors shown are those in which the error ranges associated with the three means for a given groove width are at least partially nonoverlapping. The ranges of all other triads overlap entirely. The data clearly indicate that selective adaptation has no systematic effect of any importance on the tactual perception of surface roughness.

Additional pilot work performed with the authors serving as subjects extends the generality of these findings to include paper-covered, abrasive surfaces. It was shown that perceived roughness of these surfaces was virtually unaffected by selective vibrotactile adaptation.

\section{GENERAL DISCUSSION}

The results of Experiments 1 and 2 strongly support the prediction made by the Taylor and Lederman (1975) model of roughness, whereas they fail to support Katz's (1925) interpretation. It was argued that if the sense of
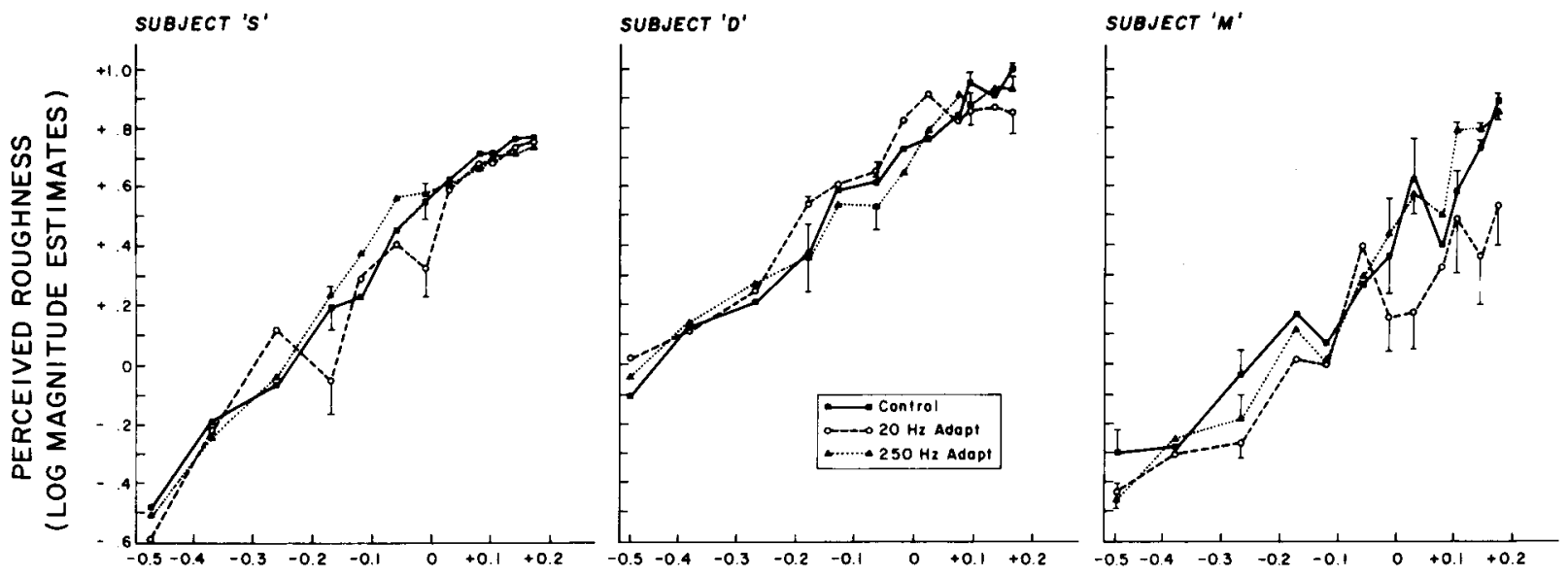

LOG GROOVE WIDTH $(\mathrm{mm})$

Figure 2. Experiment 2: Perceived roughness as a function of groove width and adaptation state (static, $20 \mathrm{~Hz}$, and $250 \mathrm{~Hz}$ ). The adapting intensities are the same as those described in the caption for Figure 1. For discussion of standard errors, see text. 
vibration does underlie the perception of roughness, then a procedure that affects the perception of vibrotactile stimulation should also affect the perception of surface roughness.

In Experiment 1, it was demonstrated that selective vibrotactile adaptation differentially affects the magnitude estimation of low- and high-frequency test stimuli. When a low- (or high-) frequency adapting stimulus is applied to the fingertip, the vibratory magnitude perceived for test stimuli of the same frequency is greatly reduced compared with corresponding estimates of the high- (or low-) frequency test stimuli. To our knowledge, selective vibrotactile adaptation effects have not previously been demonstrated at suprathreshold levels using a magnitude estimation procedure. It also should be pointed out that, since Katz (1923, cited in Geldard, 1940) believed the sense of vibration, unlike that of pressure, could not be adapted, he would not have predicted the findings of Experiment 1.

In Experiment 2, it was shown that the same conditions of selective vibrotactile adaptation had essentially no effect on the tactual perception of surface roughness. Such evidence therefore provides additional psychophysical support against the claim that the sense of vibration constitutes the sine qua non of roughness perception.

Some concern has been expressed by one of the reviewers concerning the lack of control over depth of penetration. Depth of penetration is an important variable in making vibrotactile threshold measurements (e.g., Craig \& Sherrick, 1969). However, we would argue that this lack of control does not undermine the validity of the results obtained in Experiments 1 and 2. Regardless of either systematic or unsystematic variation in the adapting stimuli produced, the same method was used in both experiments. Yet with the same stimulus conditions, vibrotactile adaptation strongly affected the perception of supraliminal vibration, while roughness was relatively unaffected. The effects were very strong, consistent, and interpretable. We therefore believe that our conclusions are fully justified despite the lack of control over depth of penetration.

Taylor and Lederman's model of roughness (1975) focuses on the intensive/spatial aspects of skin deformation. The model proposes three deformation parameters that best predict known psychophysical data on the roughness of linear gratings (e.g., Lederman, 1974). One is the depth to which the finger penetrates the grooves (mainly determined by the spacing among the elements which form the invariant spatial pattern, and by the applied force). The other two aspects of skin deformation that the model suggests are important (and possibly better) predictors of perceived roughness are two measures of the cross-sectional area (volume is the more appropriate measure) of skin penetrating the tiny grooves summed across the entire fingertip. None of these factors depends upon the dynamic aspects of vibration, for example, temporal pulse frequency and rate of skin displacement.
The perception of surface texture is clearly a multidimensional task involving spatial, temporal, and intensive properties, for example, roughness, spatial density, bumpiness, jaggedness, oiliness, stickiness, and slipperiness. There are no doubt a number of different neural codes available for assessing such textural properties. Is it possible to link the roughness percept to activity in any of the various mechanoreceptor populations of human glabrous skin? Unfortunately, little yet is known about this topic, and consequently, the following discussion must be considered speculative.

Knibestol and Vallbo (1970) have identified four functionally distinct types of mechanoreceptors in the glabrous skin of the human hand. Two rapidly adapting types are the PC units and the RA units (presumed to terminate in Meissner corpuscles, and likely to be a major determinant of the NP response). The current data suggest that neither the PC nor the RA units, which are maximally tuned to low-frequency vibration (Talbot et al., 1968), contribute in any obvious way to the perception of roughness. Knibestol and Vallbo (1970) also discuss two types of slowly adapting units. These, too, may contribute to the response of Verrillo's NP system. Units of one type, known as SAI units, are believed to terminate in Merkel cell neurite complexes, situated on the deep end of the intermediate ridges that project into the dermis. Units of the other type, SAII units, are presumed to end in Ruffini cylinders.

While the PC and RA units may not code roughness perception, it is possible that the SA units perform this function, as suggested by Lederman (1978a) and Vierck (1979). Lederman (1978a) speculated on the role of SAI units on the basis of their potential for being stimulated by shear and normal forces applied to the free-moving intermediate ridges, which, in turn, might function as microlever systems. However, the possibility that SAII units may code shear forces applied to the skin (Johansson, 1978) suggests the further possibility that they may play a role in coding the effects of shear on the perception of roughness (Lederman, 1978a). Vierck (1979) has suggested that SAI and SAII units may contribute to the coding of texture in cat; he demonstrated a strong preferential sensitivity for punctate stimulation and edges presented to a portion of the receptive fields of these units, as compared with a smooth disk presented to the entire field.

Vierck's findings may be directly relevant to the current studies. As the contactor in the current study was a large, smooth disk, the SA units might have been considerably less affected than the RA or PC units. And, because they respond well to points and edges, it is possible that the SA units coded roughness in the selective adaptation experiments. If this interpretation proves correct, then, to the extent that SA units are a part of the NP system, the NP system becomes implicated in tactual roughness perception. Further research on this interpretation is planned.

Also relevant to the psychophysical work on human roughness perception is Vierck's report that an increase 
in skin indentation was coded by individual fibers in terms of the rate of firing. Indentation is one of the three skin deformation parameters that Taylor and Lederman's model suggests may determine human roughness perception. It is not clear what neural code(s) might represent the other two deformation parameters in the model, but it seems evident that they must be represented in the spatially distributed population of mechanoreceptors and not in the response of individual fibers.

Recently, there have appeared some excellent sensory neurophysiological studies which have examined several coding mechanisms available to the monkey when textured surfaces are presented. For example, DarianSmith and his colleagues (Darian-Smith, Davidson, \& Johnson, 1980; Darian-Smith \& Oke, 1980) reported a series of elegant experiments on glabrous skin of monkey. They examined responses of QA (quickly adapting afferents, also referred to as RA units), SA (not further differentiated into SAI and SAII units), and PC units to very finely spaced gratings and two-dimensional dot patterns. All receptive fields were in the fingertips. The surfaces were moved at specified rates and forces across the skin. Their work indicated that the stimulus temporal frequency (i.e., spatial frequency of the grating $X$ velocity of the moving surface) is coded in terms of the response of single units. The three mechanoreceptor populations coded different portions of the stimulus temporal frequency range produced with the gratings: SA units, $20-60 \mathrm{~Hz} ; \mathrm{QA}, 60-200 \mathrm{~Hz}$; and PC units, $100-300 \mathrm{~Hz}$. Unambiguous information concerning spatial frequency and stimulus velocity is not available in the response of any single unit. Thus, it would appear necessary to propose an additional stage in which the responses of the relevant spatially distributed population of fibers are considered. With the two-dimensional dot surfaces, the SA population appeared to offer the clearest potential for coding the very fine, invariant spatial characteristics of the surface. Phillips and Johnson (1981) have also suggested an important role for SA fibers in spatially coding very fine detail. They demonstrated that only SA afferents are capable of coding the fine spatial detail in gratings with fundamental spatial periods $\leqslant 3 \mathrm{~mm}$. The response of RA and PC units showed no such corresponding spatial modulation. All of the gratings used in the current experiments were $\leqslant 3 \mathrm{~mm}$ in spatial period. Thus, it is possible that, provided the SA units were less adapted than either RA or PC units, a spatial code of the invariant spatial pattern would be available, at least for gratings with spatial periods above about $2 \mathrm{~mm}$.

Johnson and Davidson (1981) also expanded on the different neural codes available for differentiating dot arrays with spatial periods $>2$ and $<2 \mathrm{~mm}$. The relative response profiles of the three main afferent populations seem to be most important in providing nonspatial codes for discriminating finely textured surfaces.

Finally, we should not overlook the potential contri- bution of "free," unencapsulated, nerve endings to the perception of roughness. Such fibers are known to respond to many different kinds of physical stimulation, including, for example, light touch.

Which of the various neural codes available are actually used by the brain in interpreting surface roughness? The psychophysical studies by Lederman and her associates (e.g., Lederman, 1973, 1974, Note 1; Lederman \& Taylor, 1972) suggest that the codes associated with temporal stimulus frequency and invariant spatial period of the surface, although available, are not the most important. Rather, the work to date suggests that codes associated with interelement spacing and the forces applied to the skin are of greatest significance in human roughness perception.

\section{REFERENCE NOTE}

1. Lederman, S. J. Spatial and temporal determinants of tactual roughness perception. Manuscript submitted for publication, 1982.

\section{REFERENCES}

Capraro, A., Verrillo, R. T., \& Zwislocki, J. Psychophysical evidence for a triplex system of cutaneous mechanoreception. Sensory Processes, 1979, 3, 334-352.

Craia, J., \& Sherrick, C. The role of skin coupling in the determination of vibrotactile spatial summation. Perception \& Psychophysics, 1969, 6, 97-101.

Darian-Smith, I., \& Oke, L. Peripheral neural representation of the spatial frequency of a grating moving at different velocities across the monkey's fingerpad. Journal of Physiology, 1980, 309, 117-133.

Darian-Smith, I., Davidson, D., \& Johnson, K. Peripheral neural representation of the two spatial dimensions of a textured surface moving across the monkey's finger pad. Journal of Physiology, 1980, 309, 135-146.

Geldard, F. The perception of mechanical vibration: IV. Is there a separate "vibratory sense"'? Journal of General Psychology, 1940, 22, 291-308.

Gescheider, G. A., Frisina, R. D., \& Verrillo, R. T. Selective adaptation of vibrotactile thresholds. Sensory Processes, 1979, 3, 37-48.

HAHN, J. Vibrotactile adaptation and recovery measured by two methods. Journal of Experimental Psychology, 1966, 71, 655-658.

Johansson, R. S. Tactile sensibility in the human hand: Receptive field characteristics of mechanoreceptive units in the glabrous skin area. Journal of Physiology, 1978, 281, 101123.

Johnson, K., \& DAvidson, T. A somesthetic coding mechanism for texture like color coding in vision. Society for Neuroscience Abstracts, 1981.

Katz, D. Der Aufbau der Tastwelt. Zeitschrift für Psychologie, Leipzig: Barth, 1925.

KNibestol, M., \& VAlLbo, A. Single unit analysis of mechanoreceptor activity from the human glabrous skin. Acta Physiologica Scandinavica, 1970, 80, 178-195.

Krueger, L. David Katz's Der Aufbau der Tastwelt (The world of touch): A synopsis. Perception \& Psychophysics, 1970, 7, 337-341.

KRUEGER, L. Tactual perception in historical perspective: David Katz's world of touch. In W. Schiff \& E. Foulke (Eds.), Tactual perception: A sourcebook. New York: Cambridge University Press, 1981.

Labs, S. M., Gescheider, G. A., Fay, R. R., \& Lyons, C. H. 
Psychophysical tuning curves in vibrotaction. Sensory Process, 1978, 2, 231-247.

LEDERMAN, S. J. The perception of roughness by touch. Unpublished doctoral dissertation, University of Toronto, 1973.

LeDERman, S. J. Tactile roughness of grooved surfaces: The touching process and effects of macro- and microsurface structure. Perception \& Psychophysics, 1974, 16, 385-395.

Lederman, S. J. Heightening tactile impressions of surface texture. In G. Gordon (Ed.), Active touch: The mechanism of recognition of objects by manipulation. A multidisciplinary approach. Oxford: Pergamon, 1978. (a)

Lederman, S. J. "Improving one's touch" ... and more. Perception \& Psychophysics, 1978, 24, 154-160. (b)

Lederman, S. J., \& Taylor, M. M. Fingertip force, surface geometry and the perception of roughness by active touch. Perception \& Psychophysics, 1972, 12, 401-408.

Phillips, J., \& Johnson, K. Tactile spatial resolution: II. Neural representation of bars, edges and gratings in monkey primary afferents. Journal of Neurophysiology, 1981, 46, 1204-1225.

Talbot, W. H., Darian-Smith, I., Kornhuber, H., \& Mountcastle, V. The sense of flutter-vibration: Comparison of the human capacity with response patterns of mechanoreceptive afferents from the monkey hand. Journal of Neurophysiology, 1968, 31, 301-334.

TAYLOR, M. M., \& Lederman, S. J. Tactile roughness of grooved surfaces: A model and the effect of friction. Perception \& Psychophysics, 1975, 17, 28-36.

VERRILLO, R. T. A duplex mechanism of mechanoreception. In D. Kenshalo (Ed.), The skin senses. Springfield: Thomas, 1968.

Verrililo, R. T., \& Gescheider, G. A. Enhancement and summation in the perception of two successive vibrotactile stimuli. Perception \& Psychophysics, 1975, 18, 128-136.

Verrillo, R. T., \& Gescheider, G. A. Effect of prior stimulation on vibrotactile thresholds. Sensory Processes, 1977, 1, 292-300.

VIERCK, C. J., JR. Comparisons of punctate, edge and surface stimulation of peripheral, slowly-adapting, cutaneous, afferent units of cats. Brain Research, 1979, 175, 155-159.

(Manuscript received December 1, 1981; revision accepted for publication May 11, 1982.) 